https://artnodes.uoc.edu

\title{
El potencial de las artes para imaginar otra educación. Aprendiendo de movimientos, posiciones y tránsitos en el caso del I. E. Pepa Colomer
}

\author{
Fernando Herraiz-García \\ Universidad de Barcelona \\ Silvia de Riba Mayoral \\ Universidad de Barcelona \\ Laura Marchena-Ricis \\ Universidad de Barcelona
}

Fecha de presentación: octubre de 2021

Fecha de aceptación: enero de 2022

Fecha de publicación: enero de 2022

\section{Cita recomendada}

Herraiz-García, Fernando; de Riba Mayoral, Silvia; Marchena-Ricis, Laura. 2022. «El potencial de las artes para imaginar otra educación. Aprendiendo de movimientos, posiciones y tránsitos en el caso del I.E. Pepa Colomer». En: Garcés, Marina (coord.). «Ecología de la imaginación». Artnodes, no. 29. UOC. [Fecha de consulta: dd/mm/aa]. https://doi.org/10.7238/artnodes.v0i29.392758

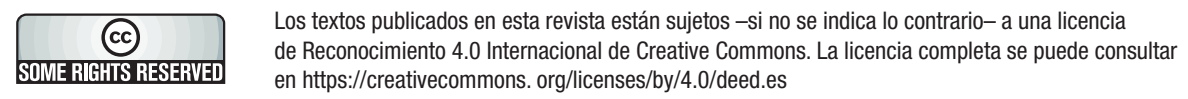

\section{Resumen}

El presente artículo invita a pensar en experiencias sobre aprender en las que la praxis artística se sumerge transversalmente dentro de un instituto-escuela, en este caso el I. E. Pepa Colomer; en primer lugar, se presenta brevemente un marco referencial que fundamenta el trabajo en esta línea, poniendo especial atención en la potencialidad de las artes para reimaginar los procesos educativos. Para ello, la noción de imaginación social propuesta por Maxine Greene $(1973,1995)$ ayuda a pensar las conexiones entre dos mundos aparentemente distantes (Martínez 2020), donde su conjunción 
permite generar espacios para la transformación y la transgresión política de la educación. De esta forma, las artes favorecen la conexión entre lo individual y lo colectivo, y habilitan capacidades agenciales (Mahlknecht 2017) tanto a estudiantes y docentes como al centro educativo en sí mismo.

En segundo lugar, planteando la necesidad de vincular la educación reglada con la sociedad y la cultura en la que se inscribe, se reflexiona en torno a las posibilidades y las tensiones de las artes para detonar modos de aprender juntas que traspasan las propias aulas y los contenidos disciplinares. Todo ello con el trabajo colaborativo de docentes del I. E. Pepa Colomer y educadoras externas vinculadas con la vida cultural de la ciudad en la que se ubica. Al respecto, la experiencia de la que damos cuenta muestra la confluencia de un proyecto artístico de centro con proyectos externos generados desde entidades culturales que invitan a imaginar modos de aprender colectivos que afectan a la vida tanto dentro como fuera de la comunidad educativa.

\title{
Palabras clave
}

educación artística; imaginación social; comunidad educativa; proyectos transversales; cultura y educación

\author{
The potential of the arts to imagine another education. Learning from movements, positions and \\ transits in the case of the Pepa Colomer School
}

\begin{abstract}
This article invites one to think about learning experiences in which artistic practice is transversally submerged within an institute-school, in this case that of Pepa Colomer School; firstly, a referential framework is briefly presented which lays the basis for the work in this vein, particularly highlighting the potential of the arts when reimagining educational processes. For this, the notion of social imagination proposed by Maxine Greene $(1973,1995)$ helps one to think about the connections between two apparently distant worlds (Martínez 2020), where their conjunction allows for the generation of spaces for political transgression and transformation of education. In this way, the arts favour the connection between the individual and the collective, facilitating agential capacities (Mahlknecht 2017) for students, teachers, and the education centre itself.

Secondly, suggesting the need to link regulated education with the society and culture in which it exists is reflected in the possibilities and tensions of the arts in order to trigger ways of learning together which transcend classrooms and disciplinary content. All of this with the collaborative work of the teachers at the Pepa Colomer School and external educators linked with the cultural life of the city in which it is located. In this regard, the experience we remark upon displays the confluence of a Central Artistic Project with external projects generated by cultural entities, which invite one to imagine collective ways to learn that affect life both in and out of the education community.
\end{abstract}

\section{Keywords}

artistic education; social imagination; education community; transversal projects; culture and education 


\section{artnodes}

https://artnodes.uoc.edu

El potencial de las artes para imaginar otra educación. Aprendiendo de movimientos, posiciones y tránsitos en el caso del I. E. Pepa Colomer

\section{Una introducción}

Ya Fernando Hernández $(1995,35)$, tras la reforma educativa implementada en el Estado español en la década de los noventa, reclamaba la figura de una ${ }^{1}$ especialista en artes que colaborase en el desarrollo de la cultura artística dentro de las escuelas e institutos. Esta demanda buscaba colocar las artes en un lugar relevante fundamentando formas de conocimiento que tradicionalmente habían quedado relegadas a un segundo plano en el currículum. Después de décadas de trabajo en docencia e investigación, el campo de estudio de la educación artística ha ido transitando por perspectivas críticas y reflexivas, posibilitando su expansión dentro de la vida escolar; y es que, cada vez más, las artes han venido asumiendo un papel que, atravesando lo disciplinar, crece enredado dentro de otras áreas de conocimiento.

Es en la noción de imaginación donde se encuentra un resquicio con el que seguir pensando en la potencialidad de las artes en educación. En esta línea, en 2017 se desarrolló en el Macba el seminario PEl Obert Aprender a imaginarse. Sobre la pedagogía y la emancipación; en dicho seminario y la publicación que derivó de este se colocó el foco en el papel de la imaginación como prácticas de transgresión y capacidad crítica a la hora de conectar elementos distantes entre sí (Martínez 2020, 13); se trataba de imaginar puentes entre el museo y la escuela como espacios y tiempos de emancipación social.

Con esta premisa y desde estos marcos, el texto busca acercarse a un centro educativo con la intención de imaginar conexiones supuestamente distantes entre la vida educativa y los proyectos culturales que se venían implementando en la ciudad; en este caso, se indagó cómo se vinculaba el Instituto-Escuela Pepa Colomer (a partir de ahora I. E. Pepa Colomer) con entidades culturales del contexto social en el que estaba situado, El Prat de Llobregat, una ciudad próxima a Barcelona.

El trabajo desarrollado en este centro era de interés, principalmente, por dos razones: primero, porque de manera regular venía desarrollando proyectos tanto externos como internos basados en las artes, donde la figura de un especialista acompañaba los procesos pedagógicos derivados de estos; $y$, segundo, por las particularidades que singularizaban su contexto social considerado de "alta complejidad» dada la diversidad cultural. Ciertamente, el ámbito invitaba a imaginar acciones que posibilitaban modos de vida al entrelazar lo individual y lo colectivo, enzarzando subjetividades múltiples y sus agencias.

En el pasado, fue tal la complejidad que, en junio de 2014, la escuela sufrió un cierre administrativo debido a los considerados malos resultados académicos, la conflictividad y la tendencia a la guetificación. Mientras que sindicatos del profesorado y asociaciones de familias se manifestaban públicamente en contra, el Departamento de Educación de la Generalitat de Cataluña refundó el centro como un instituto-escuela, cambiando su nombre, renovando el claustro docente y el equipo directivo, e implementando un nuevo proyecto educativo. En septiembre de ese mismo año se iniciaban las clases ya con un nuevo proyecto que, impulsado por las artes, contaba con la presencia de una especialista artística en el equipo directivo, tal como reclamaba Fernando Hernández (1995) en la década de los noventa. Esta especialista, encarnada en la figura de la jefa de estudios artísticos (en conjunción con el equipo docente y su directiva), era la que impulsaba el proyecto artístico del centro, facilitando propuestas pedagógicas críticas basadas en las artes, y posibilitando que agentes externos (instituciones, asociaciones, y entidades del campo de la cultura y las artes) entrasen en la vida escolar.

En el giro desarrollado se veía reflejada una epistemología que se acercaba a la dimensión social de la imaginación propuesta por Maxine Greene (1995), propiciando espacios para la transformación y la trasgresión política y social del currículum.

«También tenemos nuestra imaginación social: la capacidad de inventar visiones de lo que debería ser y lo que podría ser en nuestra sociedad deficiente, en las calles donde vivimos, en nuestras escuelas. Escribir sobre la imaginación social, me recuerda la declaración de Jean Paul Sartre de que "es el día en que podemos concebir un estado de cosas diferente que una nueva luz cae sobre nuestros problemas y nuestro sufrimiento y decidimos que estos son insoportables" (1956, 434-435). Es decir, reconocemos la dureza de las situaciones sólo cuando tenemos en mente otro estado de cosas en el que las cosas irían mejor. De manera similar, sólo cuando pensamos en aulas humanas y liberadoras en las que cada estudiante es reconocido y sostenido en su lucha por aprender a aprender, podemos percibir la insuficiencia de las escuelas burocratizadas y despreocupadas. $Y$ es posible que sólo entonces nos veamos obligados a elegir reparar 0 renovar» (Greene 1995, 5).

Cabe decir al respecto que la renovación del instituto-escuela comportó asumir, como rasgos identitarios del centro, cualidades que le vinculaban al entorno social y a la ciudadanía crítica, a valores relacionados con la inclusión, la ecología y la transparencia de la comunidad educativa, y a la vida emocional, respetando los ritmos de madurez propios de un aprender funcional y contextual. $Y$ lo que era más importante, se implementaban manifestaciones artísticas propias de la danza, la música, el teatro y las artes visuales.

Estas aberturas, trabajadas de manera transversal, son las que llevaron a este texto a prestar especial atención al papel de las artes y la imaginación social con la intención de saber más sobre las estrategias que lo hacían posible. Así, el presente artículo se escribe con el objetivo de conocer con mayor profundidad cómo el I. E. Pepa Colomer, junto a agentes externos, entran en un juego de conocimientos basados en dimensiones sociales de la imaginación mediante las artes; para ello, se exploran tanto las tensiones como el potencial transformador de las artes en el ámbito pedagógico desde un caso empírico.

1. El artículo está escrito en genérico femenino como resultado de la posición crítica a lo masculino heteronormativo. 


\section{Llegada al centro y las premisas de las que se partieron}

Paradójicamente, la entrada al centro se produjo dentro de un contexto de investigación que poco tenía que ver con la implementación de las artes dentro del currículum y de la vida en la comunidad educativa. Se llegó al instituto-escuela a partir del proyecto europeo MiCREATE (Migrant Children and Communities in a Transforming Europe. European project H2020-SC6-MIGRATION-2018-2019-2020 MIGRATION), ${ }^{2}$ con el que, por medio de métodos visuales, se trataba de comprender ciertos procesos migratorios de niñas y jóvenes en institutos y escuelas de España. Dada la compleja labor que en esta línea se estaba realizando en el I. E. Pepa Colomer, se presentó el proyecto hablando de la voluntad de implementar metodologías visuales de investigación en diversas sesiones con sus estudiantes (Herraiz-García y De Riba-Mayoral 2021).

El interés aumentaba a medida que la conversación divergía hacia el proyecto educativo; el equipo directivo hablaba de cómo las artes se colocaban dentro de las diversas materias, deviniendo agentes movilizadores de la vida escolar. En la curiosidad que suscitó aquella conversación está el origen del presente artículo. A partir de entonces, se empezó a recoger percepciones de estudiantes y a entablar conversaciones con el equipo directivo y con algunas docentes, desarrollando una serie de encuentros con los que abordar metodológicamente nuestro estudio.

\section{Cuestiones metodológicas}

Con el fin de desplegar una metodología que posibilitara responder a los objetivos, se consideraron las aportaciones que la perspectiva poscualitativa está brindando al ámbito de la investigación (St. Pierre 2011, Hernández y Revelles-Benavente 2019). De tal manera que la metodología está enredada irremediablemente con la ontología, la epistemología y la ética, así como con las propias investigadoras. Desde este enfoque, la metodología ya no busca encontrar descripciones de la realidad, como si esta fuera algo estanco y pasivo esperando ser descubierto; es una manera de configurar mundos y, por lo tanto, requiere un compromiso con la posibilidad de imaginar, situada y relacionalmente, otras formas de generar conocimientos (Haraway 2008). En el caso de este artículo, conocimientos que se puedan involucrar éticamente con las intersecciones entre las artes y la educación vividas en el I. E. Pepa Colomer.

Para llevar a cabo esta perspectiva metodológica, el uso de la reflexividad (Sultana 2007) constituyó una herramienta clave a través de la cual dar cuenta de las acciones investigadoras y de la relación de las investigadoras con el mundo que se exploró. Es decir, permitió empujar hacia una escritura en la que las investigadoras se relacionaban con las realidades, formando parte de estas desde una posición crítica y reflexiva, y entendiendo que los considerados datos no se pueden separar del propio pensamiento (Atkinson 2015). De este modo, la reflexividad posibilitó dialogar con las evidencias, y reflexionar sobre la propia observación (Guillemin y Gillam 2004). Con ello, este instrumento activó una ética relacional en la medida en que las voces de investigadoras, colaboradoras y académicas se entrecruzaron bajo hilos argumentativamente reflexivos y que se extienden a lo largo del texto.

\subsection{Métodos}

Desde esta perspectiva metodológica, la investigación se llevó a cabo a partir de diferentes métodos. En el campo de estudio se realizó:

- 1 encuentro inicial con el equipo directivo (26/11/2020): este permitió establecer el contacto con el centro, conocer el contexto y generar las preguntas de investigación.

- 4 focus groups con 15 estudiantes de segundo de la ESO (16/02/2021, 19/02/2021, 22/02/2021, 23/02/2021): basado en metodologías visuales, este método posibilitó un acercamiento a distintos aspectos de la vida de las estudiantes, dentro y fuera del centro educativo. Para el artículo, se seleccionaron aquellos testimonios que tenían que ver con su percepción sobre la escuela y su metodología de aprendizaje.

- 4 entrevistas estructuradas con agentes del claustro del I. E. Pepa Colomer (07/04/2021): esta herramienta metodológica se desarrolló con el fin de conversar con docentes sobre sus percepciones en torno al papel de las artes en el centro educativo. Se entrevistó a una maestra de educación infantil, una profesora de educación secundaria, la jefa de estudios artísticos y la directora del instituto-escuela; todas ellas tuvieron un papel significativo en el desarrollo de las experiencias educativas en las que el artículo se centra.

Los distintos métodos llevaron a seleccionar aquellas aportaciones relevantes de las colaboradoras con el fin de alcanzar los objetivos del estudio y, a partir del uso de la reflexividad, se pusieron en relación con el marco teórico elaborado paralelamente.

\subsection{La organización de los resultados y la discusión}

Coherente con la perspectiva anunciada al inicio del apartado, el texto que continúa se organizó de la siguiente manera:

2. El objetivo de este proyecto es potenciar los procesos de integración de niños y niñas migrantes revisando y explorando prácticas educativas y políticas. En MiCREATE participan diversas instituciones de Europa para responder a los retos educativos y políticos de la diversidad social y cultural producida por los movimientos migratorios. 


\section{artnodes}

https://artnodes.uoc.edu

El potencial de las artes para imaginar otra educación. Aprendiendo de movimientos, posiciones y tránsitos en el caso del I. E. Pepa Colomer

El apartado «Entre un contexto posibilitador y un marco de referencia en torno al arte en educación» permitió elaborar un marco teórico sobre las artes en educación que se puso en relación con las condiciones contextuales de la iniciativa del I. E. Pepa Colomer. Es decir, con las estrategias y acciones que hacían posible que las artes fueran parte de la vida del centro. Así, y a través de la reflexividad, fue posible entrelazar el contexto del centro educativo del que se da cuenta con las perspectivas teóricas que permitió fundamentar la experiencia.

En el apartado «Discusiones que confluyen entre marcos y estrategias nuevas» se encuentran los resultados y la discusión enmarañados. Es decir, hay un diálogo entre la perspectiva teórica anunciada y las visiones y aportaciones de las agentes en juego. La reflexividad posibilita los conocimientos que se buscan en el artículo.

Finalmente, el apartado «A modo de conclusión, una invitación para seguir aprendiendo a través de las artes en educación» genera intersticios entre las diferentes aportaciones del texto con el fin de responder a cómo el I. E. Pepa Colomer acogía las artes para habilitar formas de aprender basadas en dimensiones sociales de la imaginación, así como el potencial y las tensiones que dicha labor comportó.

\section{Entre un contexto posibilitador y un marco de referencia en torno al arte en educación}

La educación mediante las artes está llena de desafíos; la labor es compleja, por un lado, por la instrumentalización que sufre el sistema educativo por parte de determinadas políticas y, por otro, por las críticas que sufren dentro del contexto neoliberal actual. Críticas que, mediadas por lógicas basadas en la productividad (Hernández 2011), evidencian las dificultades que encarna la educación como dispositivo al orientar, modelar y determinar la vida en los tiempos actuales; en los espacios de negociación es donde se desarrollan los sujetos, ${ }^{3}$ que se mueven entre lo que el dispositivo predispone y los seres humanos viven.

Dentro de esta complejidad, en la noción de educación que propone Marina Garcés (2020), se encuentra una imagen de pensamiento para resistir a ciertas inercias dispuestas que inmovilizan y estancan:

«Educar es aprender a vivir juntos y aprender juntos a vivir. Siempre y cada vez. Es estar, pues, en lo inacabado que somos: abiertos, expuestos, frágiles. Por eso, educar es una práctica de la hospitalidad que tiene como misión acoger la existencia desde la necesidad de imaginar. Recibirla y al mismo tiempo dejarla ser en lo que tiene de irreductible y de desproporcionado» (Garcés 2020, 22).

Educar es una acción política que conlleva la necesidad de imaginar existencias posibles desde la incertidumbre que supone vivir en una transitoriedad $\sin$ fin, y desde la vulnerabilidad que entraña sentirse expuestos a actos que escapan a la imaginación individual. En el vaivén relacional y sus agencias se encuentra ese dejarla ser enmarañada en devenires plausibles e inesperados, fruto de procesos experiencialmente colectivos. Tener presente que «educar es aprender a vivir juntas y aprender juntas a vivir» permite guiar el texto hacia una exploración de los procesos en los cuales el I. E. Pepa Colomer trabajaba desde lo común e imagina, a partir del potencial de las artes, otras maneras de ser, estar, sentir y aprender en el mundo.

\subsection{Lugares donde mirar y ser mirados}

Aquellas miradas que ven en las artes algo con lo que mejorar la vida educativa apuntan hacia un camino que va más allá de prescripciones curriculares e índices de rendimiento basados en la productividad como dispositivo neoliberal. Al respecto, la condición posibilitadora de las artes se convierte en un agente catalizador de imaginación social cuando se consolida como un lugar direccionado ${ }^{4}$ (Ellsworth 2005, 16), y donde se favorecen encuentros imaginativos y agenciales entre subjetividades inscritas en espacios colectivos (Mahlknecht 2017, 126). Encuentros que activan lugares «entre», que hablan de esos intersticios en los que, como argumenta Fernando Hernández (2019), las miradas se expanden bidireccionalmente entre nosotras y los dispositivos visuales.

«El enfoque de educación a través de las artes presta atención no al "qué» (objetos, imágenes) 0 "cómo» (las formas en que interpretamos artísticamente lo que vemos y vivimos), sino al espacio "entre": donde lo que miramos y el cómo somos vistos por lo que miramos se convierte en un lugar para encontrarse e interactuar. Por lo tanto, en términos de educación, entiendo las artes como una intersección de posiciones en red, que permite adquirir una visión de las formas en que podemos ver las cosas en la sociedad y los efectos que ésta tiene sobre cada uno de nosotros» (Hernández 2019, 35).

Las artes permiten favorecer experiencias en las que se pueden expandir miradas conformando modos de imaginación individual y social en torno a los artefactos visuales en la medida en que, al mismo tiempo, hay una intensidad afectiva en estos encuentros:

«El arte tiene la capacidad de volver a trabajar los límites de un cuerpo. El arte puede reajustar lo que una persona es o no puede entender, producir y conectarse. Esto no quiere decir que una obra de arte cambie necesariamente a sus espectadores de formas prescritas, sino que las obras de arte pueden crear nuevas asociaciones y hábitos de agrupar emociones en torno a nuevas imágenes» (Hickey-Moody 2016, 261).

La noción de imaginación posibilita visiones y miradas de aquello de lo que debería o podría llegar a ser también dentro del arte en educación; visiones y miradas que favorecen conexiones, comprensiones y

3. Según Agamben (2011), hace una distinción entre los seres vivos y los dispositivos; entre ambas emerge un tercer tipo que denomina los sujetos, y que define como «eso que resulta de la relación cuerpo a cuerpo, por así decirlo, entre los vivientes y los dispositivos» (Agamben 2011, 258).

4. Ellsworth (2005) entiende la pedagogía de la direccionalidad como un lugar en el que los aprendizajes y el conocimiento se convierten en algo personal, donde aquello que se aprende entra en contacto con el cuerpo, los sentidos del yo propio y de los otros, y mundo social y cultural. 


\section{artnodes}

https://artnodes.uoc.edu

El potencial de las artes para imaginar otra educación. Aprendiendo de movimientos, posiciones y tránsitos en el caso del I. E. Pepa Colomer

producciones al crearse nuevos modos de aprender colectivo. Todo ello bajo una dimensión social de la imaginación que se compromete con el pensamiento crítico y la transformación social de aquellas que conviven aprendiendo juntas dentro de la comunidad escolar.

De hecho, el trabajo en esta dirección llevó a Wendi Kohli (2016), inspirado por Maxine Greene, a tratar de conectar lo estético, lo ético, lo intelectual, lo existencial y lo político a través del potencial de las artes en la educación, en su caso, colocando el foco en la formación del profesorado.

«Utilicé la «potencia de las artes, para hacer que mis estudiantes vieran las cosas de maneras nuevas, para desconfundizar la experiencia, para estar más «despiertos» (Greene 1973, 2). El curso emplea el concepto de imaginación social de Greene para profundizar y complicar la comprensión de los maestros sobre la cultura, la identidad y el plan de estudios. A través de la integración de experiencias estéticas multiculturales y una amalgama de pedagogías feministas y críticas, los estudiantes del curso experimentan el poder transformador y transgresor de la imaginación: la imaginación social» (Kolhi 2016, 21).

Tal como propone Marina Garcés (2020), aprender a vivir juntas debería activar nuevas maneras de ver, comprender e incidir a través de experiencias dinámicas e inacabadas que, desde el poder de la imaginación social, se mueven multidireccionalmente entre los intersticios del mirar y ser mirados por las imágenes visuales (Hernández 2019). En esta línea, se pueden crear experiencias transformadoras y transgresoras que interconecten lo artístico, lo intelectual, lo político, lo crítico, etc., cambiando la propia relación con el mundo y desestabilizando aquello que se da por sentado en el terreno de la educación. En tanto que somos realidades enredadas en un aprender que habla de nosotras mismas y de los mundos que configuramos y nos configuran, es importante asumir compromisos que medien en los modos de vivir imaginativamente con el fin de habilitar «una respuesta que no tenemos y que, sea cuál sea, no nos dejará iguales» (Garcés 2013, 63).

\subsection{La experiencia de un proyecto artístico de centro}

El texto se inicia señalando la importancia de la imaginación en la implementación de las artes de manera transversal en la educación, en la que es esencial la incorporación de especialistas artísticos en los centros educativos. Es por ello por lo que la incorporación de la jefa de estudios artísticos en el I. E. Pepa Colomer es especialmente significativa.

Aunque pasaron diversos profesionales de la educación desde que se impulsó el nuevo proyecto artístico de centro, actualmente una licenciada en Bellas Artes y diplomada en Magisterio es quien asume la responsabilidad. Esta figura forma parte de un ecosistema escolar que avanza hacia múltiples direcciones; entre otras funciones, facilita y acompaña la acogida de agentes externas propiciando modos de trabajar que, más colaborativos, fusionan la vida escolar dentro y fuera del centro.
En dicho ecosistema de aprender, se pudo percibir cómo el papel de las agentes externas se constituía en ejes vertebradores para el devenir del curso escolar acorde con el proyecto artístico de centro. Estas agentes solían ser profesionales especializadas en artes visuales, musicales y escénicas; como argumentó la jefa de estudios artísticos, dicha labor era importante porque tenían la misión de:

«Enriquecer nuestras estrategias docentes y proporcionar a los niños y jóvenes un abanico enorme de posibilidades para explorarse y descubrirse a sí mismos y a sus compañeros y docentes en una diversidad de propuestas que sin ellos sería impensable en un centro escolar» (jefa de estudios artísticos, I. E. Pepa Colomer).

Instituciones externas y sus proyectos entraban en convenio con el centro al impulsar acciones educativas. Sus agentes intervenían convirtiéndose en parte de la comunidad escolar y desarrollando formaciones con estudiantes y docentes. En esta última línea, eran relevantes las sesiones de trabajo dirigidas al profesorado para orientar y posibilitar sentidos artísticos a su trabajo pedagógico en las aulas.

Han sido diversos los proyectos externos que han entrado en el I. E. Pepa Colomer durante los últimos cursos: «Muse-E», desarrollado por la Fundación Yehundi Menuhi, donde se implementaron estrategias propias de la música y la danza; «Basket Beat» que, como entidad y proyecto implicado con el arte comunitario desde la educación social, realizó talleres que favorecían el crecimiento relacional aprendiendo música a través de pelotas de básquet; «IntersECCions», impulsado por el ayuntamiento de la ciudad donde se ubica el centro, y que potenciaba la relación entre educación, cultura y comunidad a partir de estrategias propias de las artes visuales, musicales y escénicas. Es en este último proyecto en el que se va a hacer hincapié.

\subsection{El programa «IntersECCions» en el I. E. Pepa Colomer a modo de ejemplo}

«IntersECCions. Educació, Cultura i Comunitat» es un programa que se viene desarrollando desde el año 2016 con el impulso de la Regidoría de Educación y Cultura de la ciudad. Su objetivo, principalmente, ha consistido en movilizar acciones que uniesen la labor de entidades culturales vinculadas al municipio con el trabajo educativo de los centros reglados del territorio. Como parte de estas sinergias, las artes han ido confluyendo como dinamizadoras de un aprendizaje con sentido comunitario. La iniciativa ha supuesto asumir el reto de que agentes culturales se convirtieran en educadoras externas en escuelas e institutos; de este modo, el proyecto ha favorecido la creación de espacios donde interseccionan acciones educativas y culturales que hasta el momento habían permanecido distantes o meramente anecdóticas. Las aberturas que se han imaginado e implementado dan otros sentidos a las experiencias vivenciales dentro y fuera del aula, dado que se sitúan con mayor fuerza dentro de la comunidad y la ciudad.

Una de las instituciones culturales con la que ha venido cohabitando desde 2016 en el I. E. Pepa Colomer ha sido la Escola d'Arts 


\section{artnodes}

https://artnodes.uoc.edu

El potencial de las artes para imaginar otra educación. Aprendiendo de movimientos, posiciones y tránsitos en el caso del I. E. Pepa Colomer

Visuals del Prat; de aquí surgen las especialistas en artes que entran en la vida del centro como educadoras externas. La colaboración ha implicado la configuración de un equipo impulsor formado por tres docentes del propio instituto-escuela y dos agentes provenientes de la Escola d'Arts Visuals.

En cada edición se ha coimaginado la acción educativa mediante un eje temático diferente que perdura durante un año académico; en el último curso, se enfocó en el centro escolar como espacio atendiendo a elementos potencialmente transformadores de este. El proyecto se llevó a cabo con alumnos de educación infantil y primero de primaria; una vez por semana, ocho grupos, sus maestras y las educadoras externas aprendían mediante procesos artísticos al reflexionar, por ejemplo, en torno al papel de la luz en los lugares escolares por los que transitaban y la capacidad de agencia que las estudiantes tenían para poder repensar y transformar la propia escuela.

La labor de «IntersECCions» tenía diversas dimensiones. Aparte de las intervenciones en las aulas, el proyecto llevó implícito un seguimiento que ha venido reuniendo a educadoras y docentes quincenalmente para valorar las acciones que se iban activando y resituar la potencialidad transformadora y transgresora de las artes en educación. De hecho -es tal el enredo-, el proyecto educativo de centro y del programa de «IntersECCions» se ha visto afectado recíprocamente más allá de los tiempos y los espacios de implementación, penetrando en el día a día del instituto-escuela, y posibilitando lo que estaba por venir dentro y fuera del proyecto. En esta dirección, entraron en discusión cuestiones vinculadas a: la significación de lo colectivo, las relaciones de responsabilidad entre iguales, el sentido de pertenencia a la comunidad, el valor del compromiso con el grupo, etc. La diversidad de posturas, trayectorias y perspectivas de los agentes implicados (profesoras, maestras, educadoras externas, estudiantes y alumnos) fueron percibidos como aspectos que enriquecían las experiencias de aprender.

\section{Discusiones que confluyen entre marcos y estrategias nuevas}

Que las escuelas e institutos son ecologías complejas no es una novedad; es sabido de los múltiples agentes que los habitan y de los marcos de referencia, conocimientos y experiencias diversas que entran en interacción en los proyectos educativos. Con esto en mente, y partiendo del caso del I. E. Pepa Colomer abordado en el anterior apartado, a continuación se exploran las coyunturas agenciales propias y singulares de la vida escolar. Es decir, cómo las distintas agentes del instituto-escuela se relacionan con aquello impulsado por el proyecto artístico de centro.

\subsection{La situación de las artes en el instituto-escuela}

A partir de las distintas conversaciones que se llevaron a cabo, se percibieron articulaciones experienciales que se relataban a través de movimientos, posiciones y tránsitos de un aprendizaje en el que, también, se vislumbraban determinadas tensiones. Al respecto, una maestra de infantil hacía ver ciertas asperezas cuando las artes en educación entraban en juego:

«Por una parte, la administración no da importancia a los efectos positivos que las artes aportan al desarrollo global de nuestros alumnos. Si fuesen más conscientes, los incluirían en los currículums y dotaría de recursos económicos para que se desarrollasen en horario escolar.

Por parte de la familia, sobre todo al principio, al creer que los chicos y chicas están perdiendo el tiempo y que no aprenden nada. Poco a poco se dan cuenta de que eso no es cierto y que les aporta beneficios.

Por parte de los docentes, porque a muchos de nosotros nos saca de nuestra zona de confort. Nos encontramos inseguros delante de los procesos creativos y sin el modelaje del experto nos cuesta aplicar estrategias dentro de nuestras aulas que hemos visto.

A los estudiantes, al principio, les parece que será fácil, que no servirá para nada... Pero durante el desarrollo de los proyectos van siendo conscientes de la concentración, atención y esfuerzo que supone» (maestra de educación infantil, I. E. Pepa Colomer).

Las palabras de la maestra hacían pensar en las miradas que situaban las artes en un lugar de bajo estatus con relación a los demás conocimientos educativos: la de la administración que no acababa de apostar por lo visual en la educación, la de aquellas familias que subestimaban la materia y veían el tiempo como un recurso escaso, la de las docentes vestida de inseguridad ante nuevos desafíos, y la de algunas estudiantes que hablaban de su poca utilidad y fácil ejecución.

La argumentación de la maestra retrataba y reproducía una inercia a la que hacía frente imaginativamente mostrando las carencias y virtudes dentro de la comunidad educativa en relación con las artes.

\subsection{Las tensiones surgidas por la multiplicidad de perspectivas}

En este maremágnum de fuerzas agenciales, se daban cuenta de algunos desplazamientos cuando un proyecto educativo basado en las artes entraba en la vida escolar; despuntando en esta línea, la directora explicó que:

«Más que tensiones, yo diría que son miradas diferentes que nos llevan a diferentes construcciones de un mismo hecho. Creo que en nuestro centro no hay tensiones, 0 al menos no en relación con las artes. Pero sí que creo que cada persona hace una interpretación diferente de todas las "cosas" (proyectos) que se realizan en función de nuestra situación/contexto personal y madurez hacia la cultura y el arte» (directora, I. E. Pepa Colomer).

En ese vivir juntas, la directora mostraba que los tránsitos de las subjetividades y sus inercias se encontraban durante un tiempo y en un espacio concreto dentro de una pluralidad de posturas y perspectivas educativas. En esta dirección, el trabajo sinérgico que emerge desde la multiplicidad es un desafío no siempre fácil de asumir; aun- 


\section{artnodes}

https://artnodes.uoc.edu

El potencial de las artes para imaginar otra educación. Aprendiendo de movimientos, posiciones y tránsitos en el caso del I. E. Pepa Colomer

que complejo, puede fluir hacia un aprender basado en la hospitalidad y en la necesidad de imaginar otras formas de vivirlo (Garcés 2020, 22).

\subsection{La falta de formación como límite}

La incursión transversal de las artes en educación es un marco experimental para vislumbrar y aprender realidades transgresoras y transformadoras; pero también puede ser vivido por el equipo docente bajo la presión y el desconcierto, en algunos casos, al no verse competente ante dinámicas que se ven abocados a implementar.

«Muchos profesores no han sido educados en los procesos artísticos ni desde nuestra vida como alumnos, ni desde la formación de Profesorado. Las dimensiones del currículo son difíciles de entender en todo el súper-abanico de significados para una persona que no ha sido formada en arte. Esta realidad conlleva tener ideas preconcebidas sobre lo que es y no es lo visual y la plástica como materia. Si hablamos de procesos artísticos y de hacerlos transversales, el reto ya es inmenso. [...] Cada maestro tiene que ser consciente de cómo se siente en torno a un contenido a transmitir y si lo que pretendemos transmitir es una manera de aprender; tenemos que ser muy conscientes del qué, del cómo y del porqué. Así pues, sólo se acompaña con interés, formación observaciones, modelado y atrevimiento, para ser los profesores que no tuvimos» (jefa de estudios artísticos, I. E. Pepa Colomer).

En un contexto educativo en el que, precisamente, la institución apostaba por el marco posibilitador de las artes en educación, era donde algunas docentes no se sentían competentes para afrontar su labor; la propia falta de formación y experiencia parecía centralizar la tensión. Las palabras de la jefa de estudios artísticos expresaban las dificultades que suponía moverse entre voluntades institucionales e informes de rendimiento escolar como dispositivos de control.

\subsection{Las miradas de las familias}

En algunos casos, la relación con las familias se tejía bajo sospecha por medio de argumentaciones que transitaban entre «creer que los chicos están perdiendo el tiempo y que no aprenden nada» y «poco a poco se dan cuenta de que eso no es cierto y que aporta beneficios». Y es que: «También es un reto saber transmitir a las familias como se puede aprender con estrategias que les son desconocidas sin que caigan en la desconfianza hacia el proyecto de escuela, transmitiendo esta a sus hijos e hijas. Qué y cómo hacer visibles estos procesos a las familias es un terreno bastante desconocido que vamos aprendiendo a partir de las necesidades que van surgiendo» (jefa de estudios artísticos, I. E. Pepa Colomer).

En esta línea, Teng Huang (2012), al evidenciar los efectos y peligros del neoliberalismo en la escuela, invitaba «a crear una nueva imaginación social en la mente de lo público», y afirmaba que «los educadores pueden necesitar redefinir la relación entre la escuela, la sociedad y los padres» (Huang 2012, 45).

Tratando de no caer en pesimismos poco fértiles ni en exultantes actos celebratorios, las tensiones que surgían eran diversas, pero, en diferente medida, habilitaban movimientos en el desarrollo de nuevas estrategias imaginativas que conectaban elementos aparentemente distantes, en este caso, tomando las artes como agente posibilitador. De algún modo, dichas tensiones configuraban algunos caminos que todavía estaban por transitar e imaginar socialmente.

\subsection{Las posibilidades de las artes en el I. E. Pepa Colomer}

A pesar de las tensiones que aparecieron, se fue consciente de los giros, desplazamientos y tránsitos al hablar con docentes y estudiantes. De las entrevistas con las docentes y educadoras externas, primeramente, se pudo constatar el desarrollo de propuestas de formación docente como, por ejemplo, los claustros de aprendizaje curricular basados en prácticas artísticas, 0 en dinámicas/rituales de aula, o la observación/ modelado entre iguales; todo ello como estrategias para la apreciación de lo artístico en los procesos de aprender. Además, el trabajo en esta dirección las llevaba a imaginar cada curso académico a través de un eje transversal de centro, donde se conectaban los distintos elementos que lo conformaban uniendo el trabajo de todas las aulas, siempre a partir de las artes como ámbito transdisciplinar.

En el I. E. Pepa Colomer, se dieron las artes como motor de acción adentrándose en prácticas pedagógicas que nunca eran iguales, siempre interactivas, siempre con la huella de la especificidad de cada grupo-clase. No había estudiantes pasivas y los espacios de clase eran un lugar de relaciones cambiantes donde, mediante labores grupales, las estudiantes trabajaban de modo autónomo y en comunidad. Con ello, parafraseando las palabras de una profesora, las artes aportaban al I. E. Pepa Colomer una educación relacionada con la empatía y la imaginación, además de ser generadora de aprendizajes reflexivos y críticos.

También tuvimos la ocasión de conversar en grupo con algunas estudiantes utilizando recursos visuales (cartografías visuales, líneas de vida, dibujos propios, tarjetas Dixit ${ }^{5}$ etc.). Entre otros ámbitos, la escucha atenta se agudizaba cuando comentaban algunas experiencias vividas a partir de proyectos implementados a través de las artes. Las siguientes argumentaciones son relevantes en esta línea:

«En la escuela tienes imaginación. Esto es la realidad y esto es lo que dibujas.» (estudiante 1, 2. ${ }^{\circ}$ ES0 en el I. E. Pepa Colomer).

«He escogido este dibujo [refiriéndose a una imagen del Dixit] porque está regando y cuando se riega, crecen. Las gotas son nuestro aprendizaje y las ramas son lo que crecemos cuando aprendemos" (estudiante 2, 2. ${ }^{\circ}$ ESO en el I. E. Pepa Colomer).

5. Se utilizaron las tarjetas del popular juego Dixit a modo de elicitación con la intención de favorecer conversaciones con imágenes, yendo más allá de entrevistas o encuestas donde la oralidad era el único elemento detonador de la deriva de pensamiento. 


\section{artnodes}

https://artnodes.uoc.edu

El potencial de las artes para imaginar otra educación. Aprendiendo de movimientos, posiciones y tránsitos en el caso del I. E. Pepa Colomer

«Yo creo que en la escuela nos enseñan de ser así [señalando al joven representado en una tarjeta del Dixit] nos hacen así [señalando a la imagen de una persona mayor]. A ser un adulto con sus ideas y sus formas de pensar y todo» (estudiante $3,2 .^{\circ}$ ESO en el I. E. Pepa Colomer).

«Aprendemos a estar con la gente y con el mundo» (estudiante $4,2{ }^{\circ}$ ESO en el I. E. Pepa Colomer).

"Aparte que te enseñan cosas, hay profes que con sus lecciones te enseñan valores, y eso es muy importante. Valores como respetar a Ios demás, ayudar siempre...» (estudiante $5,2{ }^{\circ}$ ES0 en el I. E. Pepa Colomer).

«Aprendemos de manera diferente» (estudiante 6, 2. ${ }^{\circ}$ ESO en el I. E. Pepa Colomer).

«Lo que más me gusta es que trabajamos por proyectos y aprendemos por proyectos» (estudiante 7, 2. ${ }^{\circ}$ ESO en el I. E. Pepa Colomer).

En las palabras de las estudiantes se distinguía una doble dimensión discursiva. Por un lado, se reconocían trabajando de una manera diferente, en este caso por proyectos a través de las artes; y, por otro, se les brindaba la oportunidad de aprender a ser en relación con el mundo social y cultural, y a madurar imaginando otras realidades.

Mientras tanto, todo ello permitía conocer mejor cómo se enlazaban ciertos marcos pedagógicos con prácticas educativas en los que, como explicaba la directora, poco a poco las artes estaban transformando la vida escolar. Esto parecía hacerse más evidente cuando proyectos externos entraban en juego en la cotidianidad de las aulas.

\section{A modo de conclusión, una invitación para seguir aprendiendo a través de las artes en educación}

El paso como investigadoras por el I. E. Pepa Colomer se vivió con la curiosidad y la incertidumbre de aquellas que se enfrentan a lo imprevisto de la vida escolar de un centro. Querer saber más sobre las experiencias educativas basadas en las artes y cómo estas abrían vías hacia una imaginación social llevó a construir una mirada que se movía entre aulas y pasillos, entre el instituto-escuela y las entidades culturales, entre docentes y educadoras externas, entre voluntades y tensiones, entre inercias y resistencias, entre estrategias artísticas y metodologías educativas, entre imaginaciones y transformaciones, entre modos de vivir y fuerzas transgresoras, etc. Sin duda, se dieron acciones que creaban espacios alternativos de ser y de devenir en el mundo, cambiando el registro de la realidad de forma imaginativa (0'Sullivan 2001). Y esto sucedía desde impulsiones institucionales que invitaban a trabajar juntas a docentes, estudiantes y educadoras externas, vinculando la vida propia del instituto-escuela con la de las entidades culturales de la ciudad. De modo bidireccional, a través de las artes y su potencial imaginativo, se comprendió cómo lo escolar se desprendía del encorsetamiento curricular, y lo local de la ciudad se extendía en la vida del aula. Y es que, tal como decía Marina Garcés (2013), se asumieron dinámicas que verdaderamente afectaron a las relaciones con el mundo y con las otras.

Conocer con mayor profundidad lo sucedido en el I. E. Pepa Colomer permite pensar en el papel de las artes en educación desde fuera y dentro de la comunidad educativa. Es sabido que los movimientos son lentos y su expansión necesita tiempo para ir calando y sentando las bases que habitan la experiencia, por un lado, por la complejidad de la contextualidad del devenir en educación y, por otro, por la potencialidad que comporta imaginar otra manera de estar, pensar y hacer que esté atenta a la diversa eventualidad del día a día en las aulas.

Ciertamente, se aprendió de las aberturas que rompían con tradiciones e inercias, de la labor encomiable de los equipos de trabajo y del gran esfuerzo personal y profesional que supuso converger la sociedad, la cultura y la vida escolar. Sin embargo, tratar de ser críticas y reflexivas en torno a lo que se vivió proyecta un sentimiento de extrañeza dado que se confluía entre «brechas» en educación, en esta ocasión -paradójicamente-, propiciadas por conjunciones institucionales. En este sentido, también se comprendió que:

«La educación, pues, somete y libera de manera internamente antagónica y contradictoria por una razón muy simple: la cultura es un sistema que liga y desliga al mismo tiempo. Vincula y da las herramientas para recrear estos vínculos. Para reproducirlos y para deshacerlos y transformarlos. Estructura y muestra los límites de cada sistema. En definitiva, obliga y hace libre al mismo tiempo. Hablar de pedagogía emancipatoria es entrar en este antagonismo y en esta contradicción. Trabajar sus tensiones desde dentro y de manera situada» (Garcés 2020, 24).

El sentimiento de extrañeza llevaba en cierta medida a distanciamientos y a imaginaciones plausibles a través de la transitoriedad de la labor, más corporizada, situada, eventual y afectiva, que cobraba sentido al desplazarse a través de constantes movimientos irregulares, más allá de la transformación social enunciada por la institución. Es por ello por lo que no se puede obviar que la cultura y la educación son dispositivos que, como dice Agamben (2011) ${ }^{6}$ siguiendo a Foucault, conjuntan, orientan, median las conductas, las opiniones y los discursos de los sujetos:

«Foucault ha mostrado, asimismo, cómo en una sociedad disciplinaria los dispositivos aluden, a través de una serie de prácticas y de discursos, de saberes y de ejercicios, a la creación de cuerpos dóciles pero libres, que asumen su identidad y su libertad de sujetos en el proceso mismo de su asubjetivación. De esta manera, el dispositivo, antes de

6. Agamben (2011) hace una genealogía en torno a la noción de dispositivo, en la cual, a través de Hegel distingue entre el poder exterior (basado en reglas de ritos e instituciones) y el sistema de creencias y sentimientos de los sujetos. Más que reconciliar lo problemático de la relación que plantea Hegel, Foucault trata de comprender los modos de actuar de los dispositivos en el interior de las relaciones dentro de los juegos de poder. 


\section{artnodes}

https://artnodes.uoc.edu

El potencial de las artes para imaginar otra educación. Aprendiendo de movimientos, posiciones y tránsitos en el caso del I. E. Pepa Colomer

todo, es una máquina que produce subjetivaciones y, por ello, también es una máquina de gobierno» (Agamben 2011, 262).

La experiencia fue vivida como una invitación a la transformación que, antagónica y contradictoria, medió en la subjetividad de aquellas que a través de las artes experimentaron la dispersión ligada y desligada de la cultura y la sociedad, en los cuerpos dóciles pero libres dentro de la propia escuela. Es por ello por lo que, del mismo modo que se piensa sobre la predisposición del sujeto en el currículum, también se deberían cuestionar las subjetividades que inspiran la expansión de la cultura dentro de la comunidad educativa, así como las configuraciones que se ven relegadas a permanecer en los márgenes. Del estudio se desprende una preocupación en torno a la instrumentalización de las artes en educación que conforman realidades concretas bajo políticas que, a pesar de los esfuerzos y los compromisos asumidos, siguen precarizando ciertos sectores culturales y artísticos a la sombra de una supuesta transformación social que suele estar alejada de aquellos que tienen que vivirla.

Por este motivo, se concluye reiterando el carácter político de la educación y la necesidad de seguir desarrollando brechas críticas para reescribir lo que podría significar aprender a vivir juntas desde lo imaginativo. Parece que, solo así, podemos empezar a transformar lo impensable.

\section{Referencias bibliográficas}

Agamben, Giogio. «¿Qué es un dispositivo?». Sociológicas, no.73 (2011): 249-264.

Atkinson, Dennis. "The adventure of pedagogy, learning and the not-known». Subjectivity, no.8 (2015): 43-56. D0l: https://doi. org/10.1057/sub.2014.22.

Ellsworth, Elisabeth. Posiciones en la enseñanza. Diferencia, pedagogía y el poder de la direccionalidad. Madrid: Ediciones Akal, 2005.

Haraway, Donna. When the Species Meet. Minneapolis: University of Minnesota Press, 2008.

Hernández, Fernando. «Diseño curricular en Educación Visual y Plástica». Revista Interuniversitaria de formación del profesorado, no.24 (1995): 21-37.

Hernández, Fernando. coord. Pensar la relación pedagógica en la universidad desde el encuentro entre sujetos, deseos y saberes. Barcelona: Dipòsit Digital Universitat de Barcelona, 2011. http://hdl. handle.net/2445/20946. Consultado el 15 de abril de 2018.

Hernández, Fernando. «Hibridar las artes y la educación para favorecer la conciencia imaginativa». Revista GEARTE, no.6 (2019): 31-42. DOl: http://dx.doi.org/10.22456/2357-9854.92168.

Hernández, Fernando y Revelles-Benavente, Beatriz. «La perspectiva post-cualitativa en la investigación educativa: genealogía, movimientos, posibilidades y tensiones». Educatio Siglo XXI, vol.37, no.2 (2019): 21-48. DOI: https://doi.org/10.6018/educatio.387001.
Herraiz-García, Fernando y De Riba Mayoral, Silvia. «Apuntes sobre los métodos visuales. Aperturas y tensiones en una investigación con jóvenes y sus experiencias en torno a la migración». Invisibilidades. Revista Ibero-americana de pesquisa em educação, cultura e artes, no.16 (2021): 56-65.

Hickey-Moody, Anna. «A femifesta for Posthuman Art Education: Visions and Becomings». Posthuman Research Practices in Education (2016): 258-266, editado por C.A. Taylor et al. DOl: https://doi. org/10.1057/9781137453082_16.

Huang, Teng. «Agents' social imagination: The 'invisible' hand of neoliberalism in Taiwan's curriculum reform». International Journal of Educational Development, no.32 (2012): 39-45. D0l: https://doi. org/10.1016/j.ijedudev.2010.11.006.

Garcés, Marina. Un mundo común. Barcelona: Ediciones Bellaterra, 2013.

Garcés, Marina. «El contratiempo de la emancipación». Pedagogías y emancipación (2020): 21-48. Barcelona: Arcadia-Macba.

Greene, Maxine. Teacher as Stranger: Educational Philosophy for the Modern Age. Belmont, Calif.: Wadsworth Pub. Co, 1973.

Greene, Maxine. Releasing the Imagination: Essays on Education, the Arts, and Social Change. San Francisco: Jossey-Bass Publishers, 1995.

Guillemin, Marilys y Gillam, Lynn. «Ethics, Reflexivity and Ethically Important Moments in Reseach». Qualitative Inquiry 10, no. 2: (2004): 261-280. D0l: https://doi.org/10.1177/1077800403262360.

Kohli, Wendy. «The Dialectical Imagination of Maxine Greene: Social Imagination as Critical Pedagogy». Education and Culture, vol. 32, no.1 (2016): 15-24. D0l: https://doi.org/10.5703/educationculture.32.1.15.

Mahlknecht, Barbara. «Doubt, excitement, pleasure: feminist practices of teaching and learning in art and education». Teaching Gender: Feminist Pedagogy and Responsibility in Times of Political Crisis (2017): 117-130, editado por Beatriz Revelles-Benavente and Ana M. González. London: Routledge.

Martínez, Pablo. Introducción a Pedagogías y emancipación. Barcelona: Arcadia-Macba, 2020: 9-20.

Martins, Caterina. S. «E agora, vai voltar tudo a ser como era? Por uma crítica às artes na educação». 10 × 10 Ensaios entre Arte e Educação (2017): 13-20, editado por Maria de Assis. Lisboa: Fundação Calouste Gulbenkia.

0'Sullivan, Simon. «The aesthetics of affect: Thinking art beyond representation". Angelaki, Journal of the Theoretical Humanities, vol.6, no.3 (2010): 125-135. D0l: https://doi. org/10.1080/09697250120087987.

St. Pierre, Elizabeth. «Post Qualitative Research: The Critique and the Coming After». In: N. K. Denzin e Y. S. Lincoln (eds.) The Sage Handbook of Qualitative Research (2011): 611-625. Thousand Oaks: SAGE Publications Inc.

Sultana, Farhana. «Reflexivity, positionality and participatory ethics: Negotiating fieldwork dilemmas in international research». ACME: An International E- Journal for Critical Geographies, vol.6, no. 3 (2007): 374-385. 


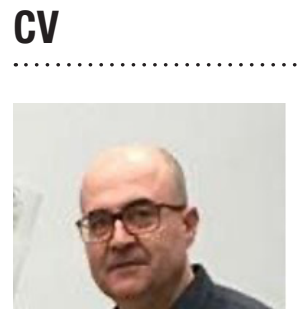

\section{Fernando Herraiz-García}

Profesor agregado

Unidad de Pedagogías Culturales. Departamento de Artes Visuales y Diseño Facultad de Bellas Artes / Universidad de Barcelona

f.herraiz@ub.edu

Profesor agregado que imparte su docencia en la Facultad de Bellas Artes (Universidad de Barcelona) dentro de la Unidad de Pedagogías Culturales; coordinó el máster de Artes Visuales y Educación: un Enfoque Construccionista desde 2018 al 2021, y está vinculado al programa de doctorado Artes y Educación desde el 2010. Interesado por temáticas en torno a la educación y los géneros, forma parte del grupo de investigación Subjetividades. Visualidades y entornos educativos contemporáneos (Esbrina), en el que ha venido trabajando en diversos proyectos sobre subjetividades y aprendizaje en diferentes entornos educativos mediante metodologías narrativas y visuales. Por otra parte, es miembro del Grupo de Innovación Docente que favorece la Indagación (Indaga-t), en el que ha participado en proyectos y propuestas que reflexionan en torno a nuevas maneras de comprender las relaciones pedagógicas en la universidad.

https://webgrec.ub.edu/webpages/000010/cat/f.herraiz.ub.edu.html http://orcid.org/0000-0001-9675-578X

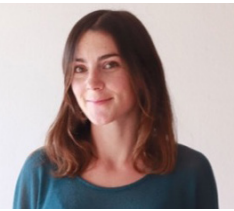

\section{Silvia de Riba Mayoral}

Doctoranda en Artes y Educación - Becada por el programa de contratación deinvestigadores predoctorales en formación PREDOCS-UB

Unidad de Pedagogías Culturales. Departamento de Artes Visuales y Diseño Facultad de Bellas Artes / Universidad de Barcelona sderiba@ub.edu

Realizadora y educadora audiovisual autónoma, y doctoranda en el programa de Artes y Educación de la Universidad de Barcelona. Realizó el grado universitario en Comunicación Audiovisual (Universidad Pompeu Fabra, Barcelona, España) y cursó el máster en Artes Visuales y Educación (Universidad de Barcelona, España). En su carrera profesional, ha trabajado en varios proyectos educativos relacionados con el audiovisual y las artes. En su tesis busca acercarse a las posibilidades pedagógicas y metodológicas que ofrecen las prácticas audiovisuales para comprometerse con las diferencias en los contextos escolares. Actualmente colabora con el grupo de investigación Subjetividades. Visualidades y entornos educativos contemporáneos (Esbrina). En concreto, está involucrada en el proyecto de investigación europeo MiCREATE (Niños y comunidades migrantes en una Europa en transformación. H2020-SC6-MIGRATION-2018-2019-2020 MIGRATION) y TRAY-AP (Trayectorias de aprendizajes de jóvenes universitarios: concepciones, estrategias, tecnologías y contextos. Proyecto I + D + i del Gobierno de España). También es asistente técnica en Matter: Journal of New Materialist Research. 


\section{artnodes}

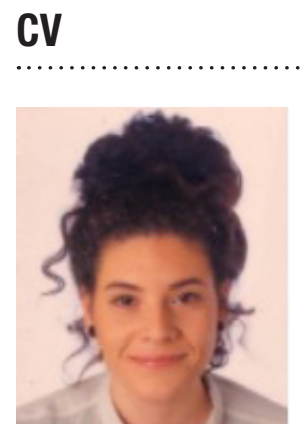

\section{Laura Marchena-Ricis}

Becaria en proyecto de investigación europeo MiCREATE (HORIZON 2020)

Beca de Colaboración en Departamentos. Departamento de Artes Visuales y Diseño

Facultad de Bellas Artes / Universidad de Barcelona

Imarchen@ucm.es

Pedagoga y actual estudiante del posgrado en Cooperación Internacional al desarrollo (Universitat Oberta de Catalunya). Realizó su grado universitario en Pedagogía por la Universidad Complutense de Madrid, y participó en el programa Erasmus+ en Sofía (Bulgaria) donde se involucró en el proyecto The Refugee Project (Cáritas) como profesora voluntaria de artes a niños y mujeres refugiados. Cursó el máster en Artes Visuales y Educación de la Universidad de Barcelona (Barcelona, España) y se incorporó como becaria en el proyecto de investigación europeo MiCREATE (Niños y comunidades migrantes en una Europa en transformación) y en el Grupo de Innovación Docente que favorece la Indagación (Indaga-t) a través de una beca de colaboración en departamentos. Actualmente estudia sobre la intersección entre las relaciones internacionales, la globalización, los estudios migratorios y la interculturalidad con los procesos artísticos. 